\title{
Ion energy distributions from laser-generated plasmas at two different intensities
}

\author{
Giovanni Ceccio ${ }^{1, *}$, Lorenzo Torrisi ${ }^{1}$, Masahiro Okamura ${ }^{2}$, Takeshi Kanesue ${ }^{2}$ and Shunsuke Ikeda $^{2}$ \\ ${ }^{1}$ Dottorato di Ricerca in Fisica-Dipartimento MIFT, V. le F.S. d'Alcontres 31, Messina 98166, Italy \\ ${ }^{2}$ Brookhaven National Laboratory, Upton, NY 11973, USA
}

\begin{abstract}
Laser-generated non-equilibrium plasmas were analyzed at Brookhaven National Laboratory (NY, USA) and MIFT Messina University (Italy). Two laser intensities of $10^{12} \mathrm{~W} / \mathrm{cm}^{2}$ and $10^{9} \mathrm{~W} / \mathrm{cm}^{2}$, have been employed to irradiate $\mathrm{Al}$ and $\mathrm{Al}$ with $\mathrm{Au}$ coating targets in high vacuum conditions. Ion energy distributions were obtained using electrostatic analyzers coupled with ion collectors. Time of flight measurements were performed by changing the laser irradiation conditions. The study was carried out to provide optimum $\mathrm{keV}$ ions injection into post acceleration systems. Possible applications will be presented.
\end{abstract}

\section{Introduction}

In order to produce high energy particles, traditional accelerators or high intensity lasers can be used. From interactions of laser with solid thin target, in high vacuum condition, it's possible to obtain energies in the order of $\mathrm{keV}$ at about $10^{10} \mathrm{~W} / \mathrm{cm}^{2}$, while using $10^{20}$ $\mathrm{W} / \mathrm{cm}^{2}$ it is possible to reach energies of MeVs [1].

The most diffused regime to induce high ion acceleration, using sub-nanosecond laser pulses interacting with thin foils, it's known as Target Normal Sheath Acceleration (TNSA), in which the plasma is produced mainly in forward direction with high energy ions above $1 \mathrm{MeV}$ per charge state [2].

Another acceleration regime is the Backward Plasma Acceleration (BPA), that generally occurs during the interaction with thick targets with low intensity lasers $\left(10^{9-13} \mathrm{~W} / \mathrm{cm}^{2}\right)$ generating high ion yields and low energetic ions, of the order of $1-10 \mathrm{keV}$ per charge state [3]. The latter can be used for the generation of plasma that find uses in several applications, for example Laser Ion Source (LIS) and post ion acceleration to generate implantable ions $[4,5]$. After the laser interaction, it is possible to observe the erosion of surface with the presence of a crater in the case of thick target and a micrometric hole in thin foils. The eroded material, converted in plasma and from which ions are produced, depends on the target composition and laser characteristics [6].

In order to study the plasma properties generated by low laser intensity and composed targets, we investigated some Al substrates targets with $\mathrm{Au}$ coating using different plasma diagnostics.

The plasma characterizations were performed using time-of-flight (TOF) techniques both at Brookhaven National Laboratory (BNL) and plasma laser laboratory of University on Messina. The interest for these plasmas comes for LIS applications in accelerator systems, such as the superconducting cyclotrons, and radio frequency quadrupole (RFQ) accelerator in which the laser-plasma produced ions can be injected.

\section{Experimental Setup}

\subsection{Laser System}

At Brookhaven National Laboratory a nanosecond $\mathrm{Nd}$ :YAG laser beam, with $10^{12} \mathrm{~W} / \mathrm{cm}^{2}$ intensity, was used at fundamental frequency with energy of $1094 \mathrm{~mJ}$ measured on the target surface, time per pulse of $6 \mathrm{~ns}$ and focalized spot of $100 \mu \mathrm{m}$ in diameter. The lasertarget incident angle was $20^{\circ}$. In University of Messina was employed also a Nd:YAG laser system at fundamental frequency, but with a laser energy of 100 $\mathrm{mJ}$ and $3 \mathrm{~ns}$ pulse duration, focalized with a spot of 1 $\mathrm{mm}$ in diameter in order to obtain a laser intensity of about $10^{9} \mathrm{~W} / \mathrm{cm}^{2}$. In both cases the interaction chamber was placed at $10^{-6}$ mbar of vacuum.

\subsection{Al coated with Au film targets}

Thin gold films on the aluminium substrates were prepared using physical vapour deposition (PVD) technique. For plasma production, we have employed a different film thicknesses, from $250 \mathrm{~nm}$ up to $750 \mathrm{~nm}$, of $\mathrm{Au}$ deposited on $\mathrm{Al}$ substrate. In addition, pure Al substrate was also prepared, in order to understand the differences with the addition of $\mathrm{Au}$ layers. The thicknesses of gold films were measured during the deposition process with a resonant quartz crystal monitor. The surface roughness, measured with a surface profiler, was very low and in the order of 1 micron. The prepared targets were mounted on a 2-axis linear stage,

\footnotetext{
Corresponding author: gceccio@unime.it
} 
moved after every laser shot; in order to irradiate only fresh surfaces in single laser pulse and high vacuum conditions.

\subsection{Faraday Cup and Ion Energy Analyser}

The plasma diagnostics in BNL are based on Faraday Cup (FC) and Ion Energy Analyser (IEA) both in Time of Flight (TOF) configuration [7]. Thanks to FC, located at $2.4 \mathrm{~m}$ away from the target, we can get the first informations on laser-generated plasma, like the maximum ion energy and the yield of plasma. The FC is located in normal direction of expansion of plasma and after the measures, can be removed to allow at the particles to travel to the IEA. The IEA is based on the electrostatic deflection of the ions, emitted from the plasma, due to an electrostatic field. IEA allows to determine the ion energy and charge state distributions by varying the voltage of the deflection plates. A symmetric potential to deflect of $90^{\circ}$ the ions towards the electron multiplier is used, according to the following relation:

$$
E / z=e k U
$$

where $E$ is the ion energy, $z$ is the charge state, $e$ is the electron charge, $k$ is the geometrical factor of IEA and $U$ is the potential gap between the plates. Only the ions with a given energy-to-charge state ratio, E/z, can pass through the IEA and arrive to the electron multiplier, thus IEA operates such as an energy filter [8].

IEA detector is placed at $1.4 \mathrm{~m}$ after the FC position for a total flight distance of $3.8 \mathrm{~m}$ from target. In Fig. 1a is plotted the experimental setup present in BNL where are indicates the laser incidence direction, the vacuum value of operation and the diagnostic position. The same diagnostics were employed in Messina with the same configuration but different distances, $0.88 \mathrm{~m}$ and $1.45 \mathrm{~m}$ for IC Ring and IEA, respectively.

The particles that pass through the IEA reaching the detector, was in an Hamamatsu R2362 Secondary Electron Multipliers (SEM). Electron multipliers are mainly used as high efficient ion detectors. Hamamatsu electron multipliers have a high gain (multiplication factor) and a low dark current, allowing operation in photon counting mode, to detect and measure extremely small low energy incoming particles. Fig. $1 \mathrm{~b}$ reports the electron emission ratio of SEM as function of ion acceleration voltage.

In Fig.1c is reported a typical IEA spectrum obtained irradiating $750 \mathrm{~nm}$ of $\mathrm{Au}$ on $\mathrm{Al} \mathrm{bulk}$; it is possible to observe several charge states of gold from 1+ up to 5+, the resolution of device allows to observe the separation between two charge states.

\section{Results}

\subsection{BNL measurements}

Fig. 2 shows the time of flight spectra of radiation emitted from laser generated plasma irradiating Al pure target and Al with Au films of different thicknesses: 250 $\mathrm{nm}, 400 \mathrm{~nm}, 500 \mathrm{~nm}$ and $750 \mathrm{~nm}$. The insert shows a zoom of the peaks of last two thicknesses $(500 \mathrm{~nm}$ and $750 \mathrm{~nm}$ of gold), the bigger quantitive of gold reduced the yield value up to the value of few $\mathrm{mV}$. All signals show a photopeak due to photoelectric effect, that gives the start time, and the ion peak located at different TOF times from which is possible to evaluate the ion energy knowing the flight distance.
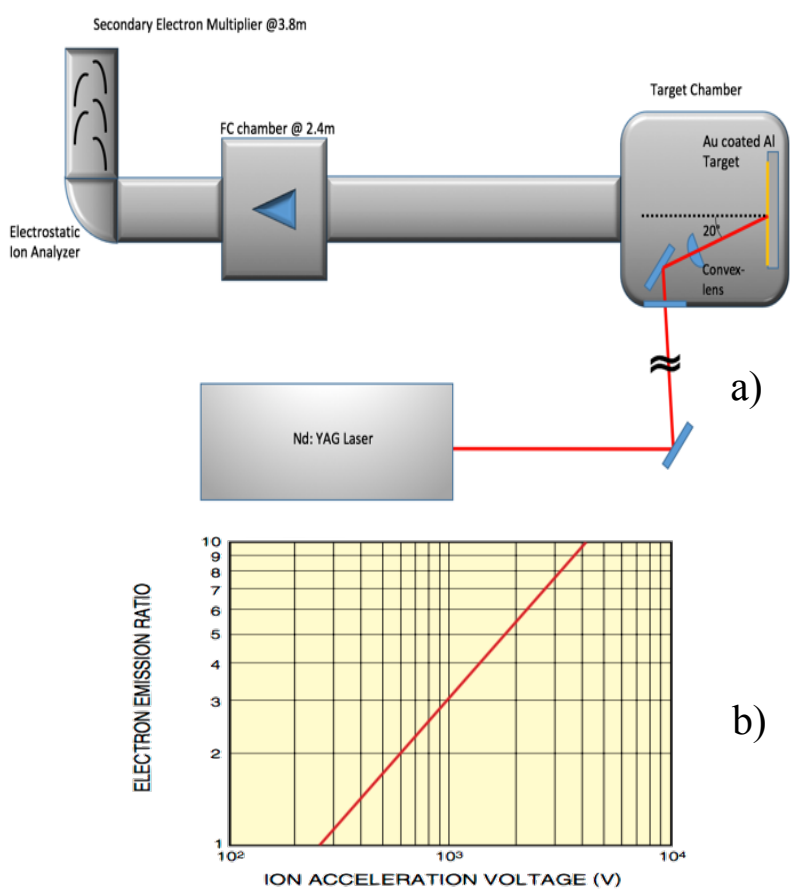

b)

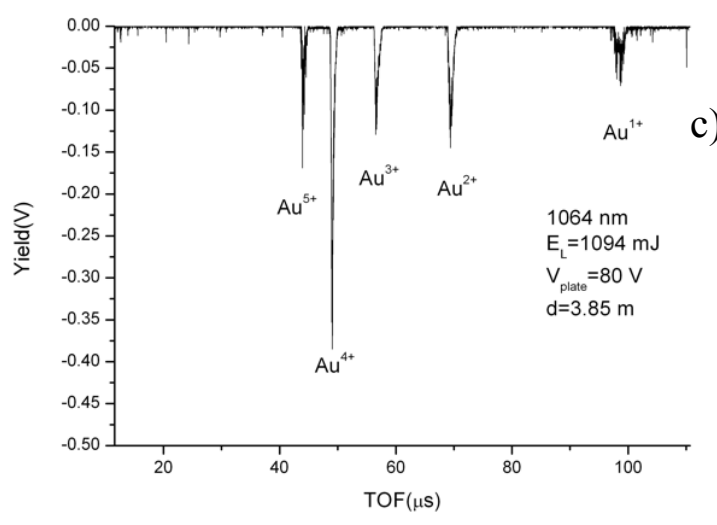

Fig. 1. Experimental setup of BNL laboratory for Plasma diagnostics. b) The Electron emission ratio for Hamamatsu SEM R2362. c) Example of IEA spectra obtained at BNL.

Observing the ions TOF values and the yields for each target it is possible to note that these values have a trend depending on the $\mathrm{Au}$ thickness. In particular the TOF value for Al pure was $5.76 \mu \mathrm{s}$, for $250 \mathrm{~nm}$ of $\mathrm{Au}$ on $\mathrm{Al}$ was $6.55 \mu \mathrm{s}$, for $400 \mathrm{~nm}$ of $\mathrm{Au}$ on $\mathrm{Al} 10.83 \mu \mathrm{s}$, for 500 $\mathrm{nm}$ of $\mathrm{Au}$ on Al $14.13 \mu \mathrm{s}$ and for $750 \mathrm{~nm}$ of $\mathrm{Au}$ on Al $14.8 \mu \mathrm{s}$. From these values, it is possible to deduce that the target with $250 \mathrm{~nm}$ of gold film has behaviour similar 
to the Al pure target but with lower energy, instead the $750 \mathrm{~nm}$ target shows a result similar to Au bulk, in terms of times of flight and yield, the 400 and $500 \mathrm{~nm}$ targets have an intermediate behaviour.

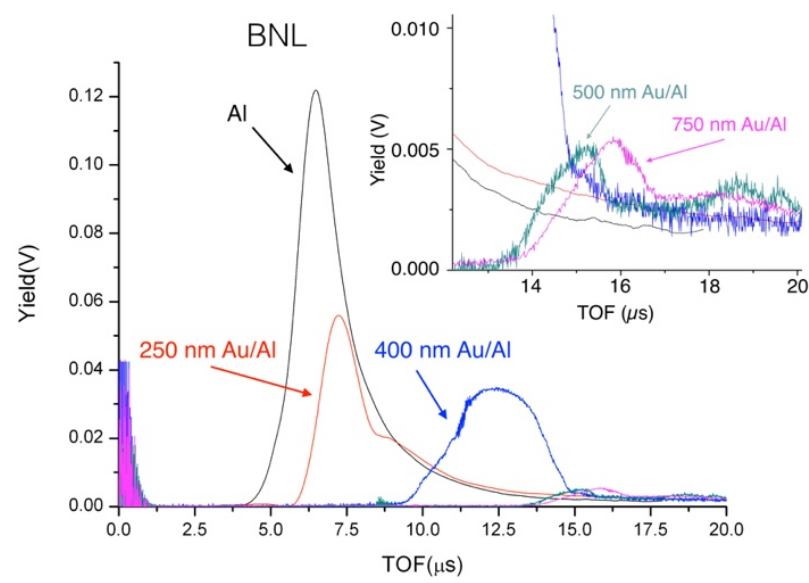

Fig. 2. ToF spectra for coated target irradiated at BNL, the reduction of energy and yield follow the thicknesses of gold films.

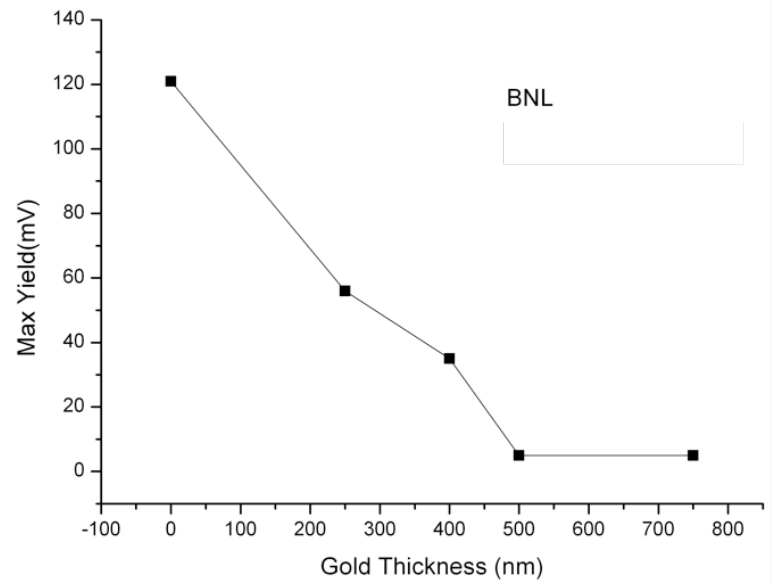

Fig. 3. Measured plasma yield with FC.

In Fig. 3 is reported the maximum yield for each target as a function of the gold thickness.

The total ion yield shows a maximum value of $120 \mathrm{mV}$ in pure $\mathrm{Al}, 56 \mathrm{mV}$ for $250 \mathrm{~nm}$ of $\mathrm{Au}$ on $\mathrm{Al}$ and decreases with the thickness until to reach the value of $5 \mathrm{mV}$ for the higher gold thickness $(750 \mathrm{~nm})$. This result shows that the $\mathrm{Al}$ ion yield are reduced when are covered by $\mathrm{Au}$ thin films, as expected.

Additional information about the maximum energy, can be obtained from TOF spectra making the deconvolution operation using the Coulomb-Boltzmann Shifted (CBS) function [9]:

$$
f(t)=A \sqrt{\left(\frac{m}{2 \pi k_{B} T}\right)^{3}} \frac{L^{4}}{t^{5}} \exp \left[-\frac{m}{2 k_{B} T}\left(\frac{L}{T}-\sqrt{\frac{\gamma k_{B} T}{m}}-\sqrt{\frac{2 z e V_{0}}{m}}\right)^{2}\right]
$$

where A represents the normalization constant, $\mathrm{m}$ the atomic mass, $\mathrm{L}$ the distance between the target and the ion collector, $\gamma$ the adiabatic coefficient (1.67 for monoatomic species), ze the ion charge, $V_{0}$ the equivalent voltage developed in the non-equilibrium plasma, and $\mathrm{k}_{\mathrm{B}} \mathrm{T}$ the equivalent plasma temperature in $(\mathrm{eV})$.

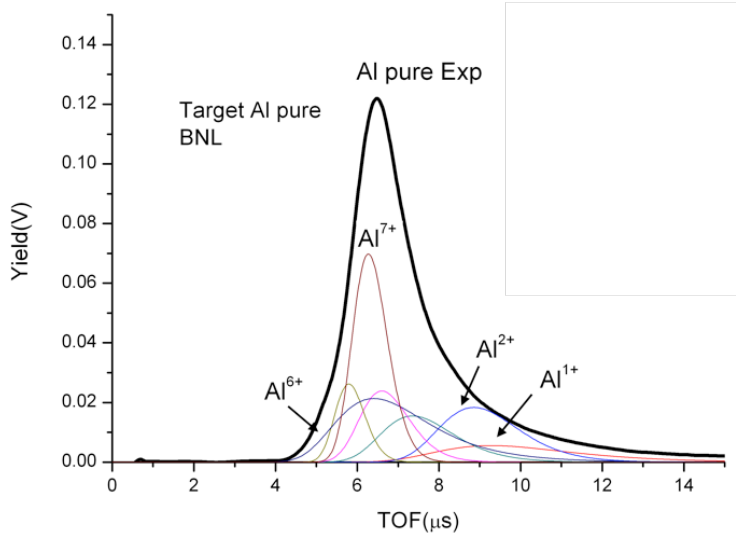

Fig. 4. Result of deconvolution on Al pure target using CBS function.

Fig. 4 reports a typical deconvolution process using the CBS function, for the pure Al spectrum. The value of 4.5 $\mu$ s corresponds to a kinetic energy of $38.5 \mathrm{keV}$ obtained from the higher charge state that in this case is $6+$.

The slower part of curve consists in lower charge states, in particular to $1+$ and $2+$. Observing the faster $\mathrm{Al}$ and $\mathrm{Au}$ ions TOF values in all targets, we obtained the values of energies reported in Fig. 5, where it is clear that the trend of ion energy is similar at the trend of the ion yield and decreases with the gold thickness from a value of $38.5 \mathrm{keV}$ to $2 \mathrm{keV}$ for Al pure target and from $51 \mathrm{keV}$ up to $28 \mathrm{keV}$ for $500 \mathrm{~nm}$ and $750 \mathrm{~nm}$ of $\mathrm{Au}$ film thickness, respectively.

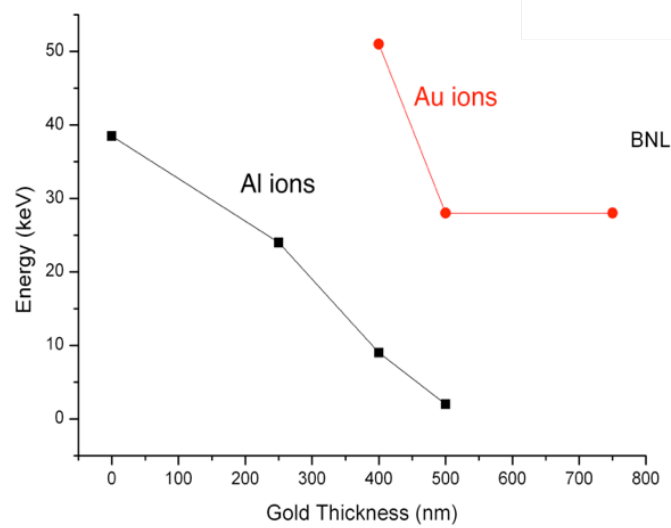

Fig. 5. Maximum energy value for $\mathrm{Al}$ and $\mathrm{Au}$ ions obtained from deconvolution of TOF spectra. 
Another parameter that change with the thickness of the gold coverage is the plasma temperature. For a calculation of the plasma temperature we have used the energy distribution obtained from IEA measurements, taking in consideration the charge states and making a fit operation using the CBS function in energy. From these deconvolutions, performed for $\mathrm{Al}$ and for $\mathrm{Au}$ in the produced plasmas, we obtained the plasma temperature for our targets reported in Fig. 6. This plot shows that the temperature is constant for $\mathrm{Al}$ pure and less than $1 \mathrm{keV}$ but for the other targets covered with $\mathrm{Au}$ films, it increases with the charge state and gold thickness reaching values up to $4.5 \mathrm{keV}$ for the charge state 5+ of $\mathrm{Au}$ with $750 \mathrm{~nm}$ of film thickness. The results demonstrate that temperature gradients characterize the produced plasma depending on the kind of irradiated target. With IEA measurements one parameter that can be possible to calculate is the ion energy acquired per charge state.

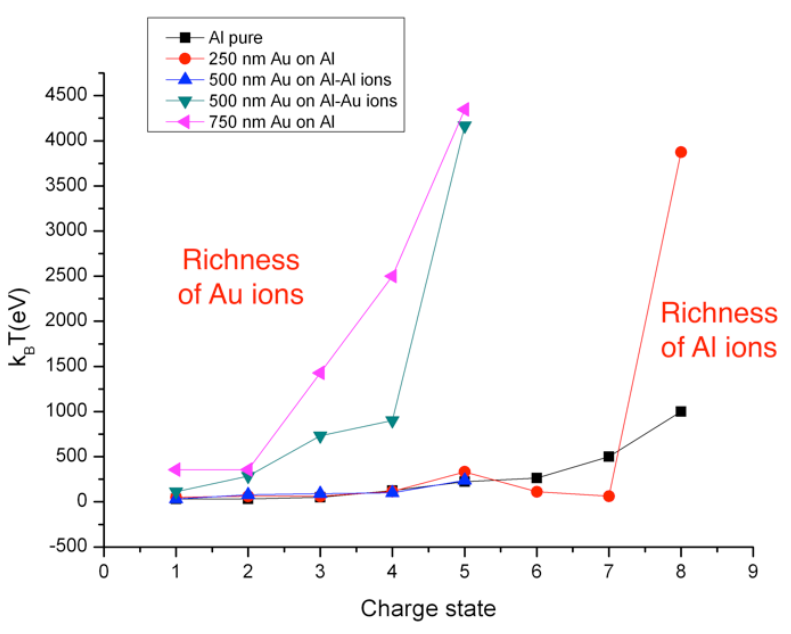

Fig. 6. Plasma temperature obtained from fit operation using CBS

\subsection{Messina measurements}

The results obtained in Messina laboratory showed similar results. The maximum peak yield and the peak energy versus the gold thickness are shown in Fig.7: full symbols indicate the results for particles energy, while the empty symbols the yield of FC signal. The yield of FC signal decreases from a value of $112 \mathrm{mV}$ for $\mathrm{Al}$ pure target up to $20 \mathrm{mV}$ for $750 \mathrm{~nm}$ of gold on Al. The ion energy increases with the film thickness reaching the values of $5.5 \mathrm{keV}$ and $1.6 \mathrm{keV}$ for $\mathrm{Au}$ and $\mathrm{Al}$ ions, respectively. Of course, the lower intensity laser reduces the charge states and the ion energy and the plasma temperature, as expected.

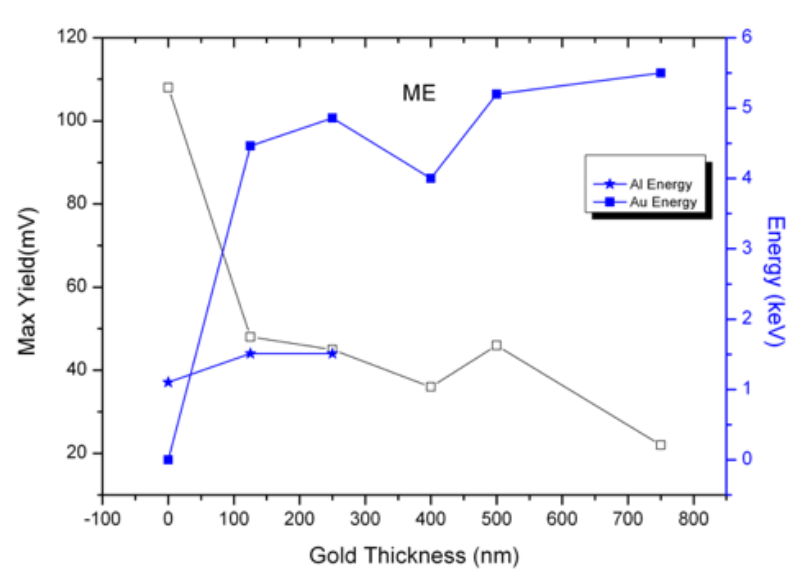

Fig. 7. Experimental results obtained in Messina

\section{Conclusions}

The reported data indicate that using a laser intensity of about $2.310^{12} \mathrm{~W} / \mathrm{cm}^{2}$ irradiating pure $\mathrm{Al}$ the plasma temperature is under $1 \mathrm{keV}$. Using the same laser intensity irradiating $750 \mathrm{~nm} \mathrm{Au} / \mathrm{Al}$ increase up to $4.5 \mathrm{keV}$, demonstrate that the increment of the electron density of the plasma, due to the presence of gold, enhances the plasma temperature. The gold in the plasma produces also an increment of the charge separation and an increment of the ion acceleration that from the $900 \mathrm{eV}$ per charge state grows up to the value of $7.5 \mathrm{keV}$ per charge state. The use of the $750 \mathrm{~nm} \mathrm{Au}$ on Al produces a plasma very rich in gold and in electrons, and very poor in Al, demonstrating that this thickness is the minimum value to produce ablation from gold similar to a bulk gold target. Using lower laser intensity of $10^{9} \mathrm{~W} / \mathrm{cm}^{2}$ the plasma yield decreases from a value of $112 \mathrm{mV}$ for pure Al up to $20 \mathrm{mV}$ with the cover of $750 \mathrm{~nm}$ of gold, with all intermediate thicknesses that follow the trend. The plasma energy increases its value with the presence of electrons from the gold films. Considering the results obtained about the charging states in the two different laboratories using two different laser intensities, we can state that at a laser intensity of $2.3 \times 10^{12} \mathrm{~W} / \mathrm{cm}^{2}$ it is possible to obtain higher charging states, for aluminium from $1+$ to $8+$ whereas for $\mathrm{Au}$ from $1+$ to $12+$, while at lower intensity the maximum charge states for both species are 3, but with more energy constants than for the high intensity. Furthermore, the studies have shown that the plasma temperature is constant for the various charge states in plasmas obtained in Messina laboratory. These obtained results suggest different applications. Direct Plasma Injection Scheme (DPIS) is a possible application where coated target can be used to produce high charge states. The DPIS in the new method combining a Laser Ion Source and a Radio Frequency Quadrupole (RFQ) Linac without a beam transport. In the DPIS, multiple charge state ions are extracted from plasma at the entrance of RFQ without selection of charge state [10-11]. In the DPIS scheme a target is located in a vacuum chamber directly connected to an RFQ Linac. In this way, even if there is an angular 
distribution of emitted ions a large fraction of plasma, it goes directly into the RFQ channel. Typically, parameters of RFQ are $100 \mathrm{MHz}$ of operating frequency and input energy of $17 \mathrm{keV} / \mathrm{amu}$. This means that our measures can be used to increasing the charge state inside the plasma and coupling with extraction voltage. Future investigation will be done to characterize the best operation frequency and the best output current beam with coated target. Using low intensity laser, it would be possible to use the coupling of an ion source that uses the coated targets with an Electron Cyclotron Resonance (ECR) that allows to enhance charge state producing ions with charge state up to $30+$ and with extraction voltage of about $20-30 \mathrm{kV}$ as performed at INFN-LNS experiments in Catania (Italy). [12]

This work was supported by the "Research and Mobility" project of Messina University No. 74893496, scientifically coordinated by Professor L. Torrisi.

\section{Bibliography}

1. L. Torrisi, NUKLEONIKA; 60(2): 207-212 (2015)

2. J. Badziak et al., Plasma Phys. Contr. Fusion 46, B541 (2004)

3. J. Badziak, P. Parys, A. B Vankov, J Wolowski, and E. Woryna, Appl. Phys. Lett. 79, 21 (2001)

4. K. Sakakibara, M. Okamura, S. Kondrashev, T. Hattori, H. Kashiwagi, T. Kanesue, Rev. Sci. Instrum 77, 03 B304 (2006)

5. K. Kondo, T. Kanesue, J. Tamura, M. Okamura, Rev. Sci. Instrum. 81, 02A511 (2010)

6. D. Margarone, L. Laska, L. Torrisi, et al., Applied Surface Science, 254, 2797-2803, (2008)

7. L. Laska et al. Czechoslovak Journal of Physics, 46, 1099-1115, (1996)

8. E. Woryna et al., Laser and Particle beams, 13, 293321, (1996)

9. L. Torrisi, Radiation Effects and Defects in Solids, 171:1-2, 34-44, (2016)

10. J. Tamura. M. Okamura, et al., Joint Accelerator Conference Website, HIAT09, 2009

11. Y. Fuwa, M. Okamura, et al., Joint Accelerator Conference Website, LINAC14, 2015

12. S. Gammino, L. Torrisi, et al,. Proceeding of EPAC 2002 